The Journal of Bone E Joint Surgery
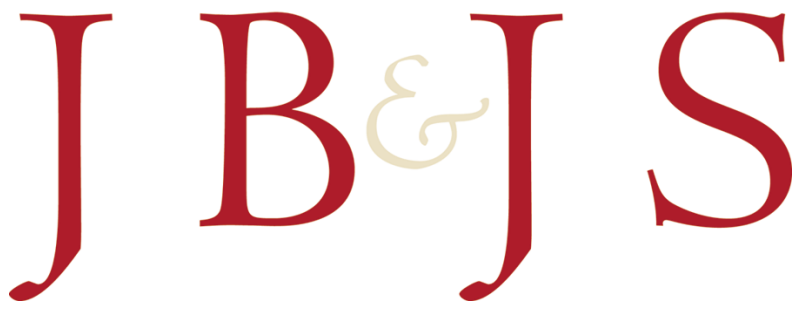

This is an enhanced PDF from The Journal of Bone and Joint Surgery

The PDF of the article you requested follows this cover page.

\title{
Emergent Management of Pelvic Ring Fractures with Use of Circumferential Compression
}

Michael Bottlang, James C. Krieg, Marcus Mohr, Tamara S. Simpson and Steven M. Madey J Bone Joint Surg Am. 2002;84:43-47.

\section{This information is current as of July 20, 2007}

Reprints and Permissions

Publisher Information
Click here to order reprints or request permission to use material from this article, or locate the article citation on jbjs.org and click on the [Reprints and Permissions] link.

The Journal of Bone and Joint Surgery

20 Pickering Street, Needham, MA 02492-3157

www.jbjs.org 


\title{
Emergent Management of Pelvic Ring Fractures with Use of Circumferential Compression
}

\author{
By Michael Bottlang, PhD, James C. KrIEG, MD, \\ Marcus Mohr, Tamara S. Simpson, MD, And STEVEn M. Madey, MD
}

\section{Introduction}

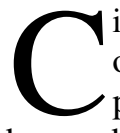

rcumferential compression of the pelvis has been recommended for emergent stabilization of open-book pelvic fractures to reduce the risk of life-threatening hemorrhage $^{1-3}$. However, little information on the application, effectiveness, and safety of this procedure is available to date ${ }^{3,4}$. We therefore investigated noninvasive stabilization of pelvic ring fractures in three cadaveric biomechanical studies. First, we established optimal application parameters for circumferential compression of open-book pelvic fractures. Second, we derived the amount of pelvic stabilization that can be achieved by application of a novel pelvic sling that provides for controlled circumferential compression. Finally, we determined whether this pelvic sling could be applied safely at the emergency scene, where the specific pelvic fracture pattern is not readily assessable. The results of this research demonstrate that circumferential compression with this noninvasive pelvic sling is an effective and safe method for reducing and stabilizing open-book pelvic fractures at the emergency scene.

\section{Methods}

A pplication Parameters: A cadaveric study was performed $A$ to determine the most effective application site and the force required to reduce open-book pelvic fractures. Partially stable and unstable open-book pelvic fractures (Young-Burgess type-II and III anteroposterior compression fractures associ- ated with a 50 and $100-\mathrm{mm}$ diastasis of the symphysis pubis, respectively) were created sequentially in seven nonembalmed human cadavers from individuals with an average age at death of $80 \pm 7$ years, an average height of $170 \pm 9 \mathrm{~cm}$, and an average weight of $74 \pm 8 \mathrm{~kg}$. An experimental pelvic sling consisting of a 50-mm wide, flexible, nonelastic belt was designed. This sling was applied at three distinct transverse levels (levels I, II, and III), extending from the symphysis pubis to the iliac crest (Fig. 2). Pelvic reduction was determined with use of a symphysis contact sensor. The quality of reduction was determined with use of three-dimensional-motion tracking sensors mounted on each hemipelvis. The required sling tension was measured with use of a load cell.

Efficacy Evaluation: The pelvic stabilization achieved with use of the noninvasive pelvic sling was compared with that achieved with use of invasive stabilization alternatives in a study of eight nonembalmed human cadavers from individuals who had an average age at death of $73 \pm 8$ years, an average height of $174 \pm 8 \mathrm{~cm}$, and an average weight of $69 \pm 17 \mathrm{~kg}$. Unstable unilateral open-book fractures (Young-Burgess typeIII anteroposterior compression fractures associated with a $100-\mathrm{mm}$ diastasis of the symphysis pubis) were created. Stabilization was provided first with use of the pelvic sling, which was applied around the greater trochanters and tensioned to $180 \mathrm{~N}$. Subsequently, stabilization was provided with use of a posterior pelvic C-clamp and an anterior external fixator
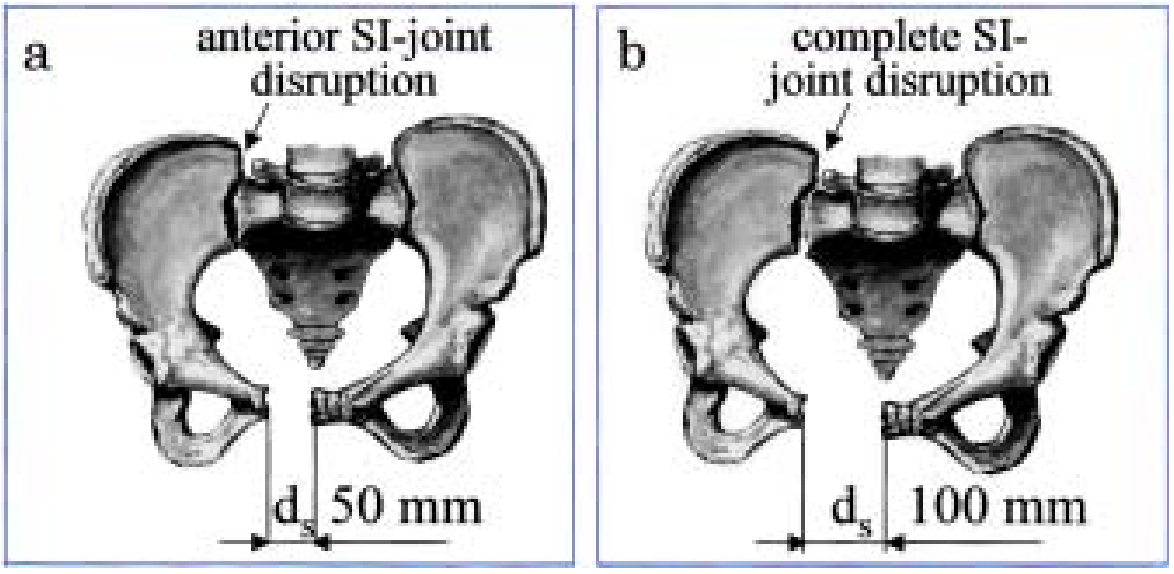

Fig. 1

Open-book pelvic fracture models. a, Unilateral partially stable Young-Burgess type-II anteroposterior compression fracture. $b$, Unilateral unstable Young-Burgess type-III anteroposterior compression fracture. 
THE JOURNAL OF BONE \& JOINT SURGERY - JBJS.ORG Volume 84-A · SuPPlement 2 - 2002

Emergent Management of Pelvic Ring Fractures With Use of Circumferential Compression

Fig. 2

Application of the pelvic sling with sling tension $\left(F_{t}\right)$ at transverse levels I, II, and III, extending from the symphysis pubis to the iliac crest.

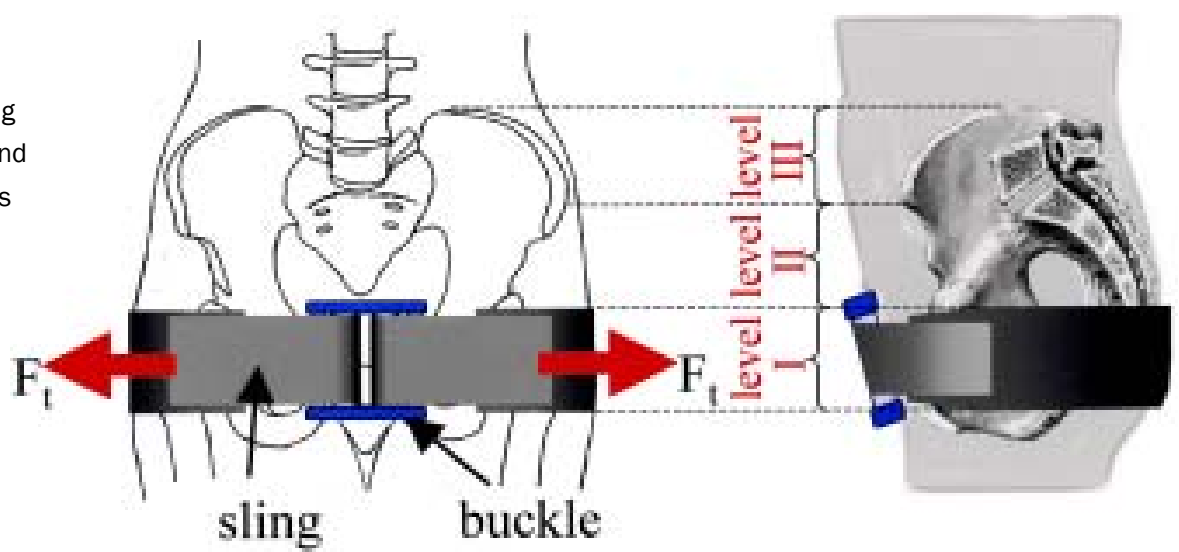

a

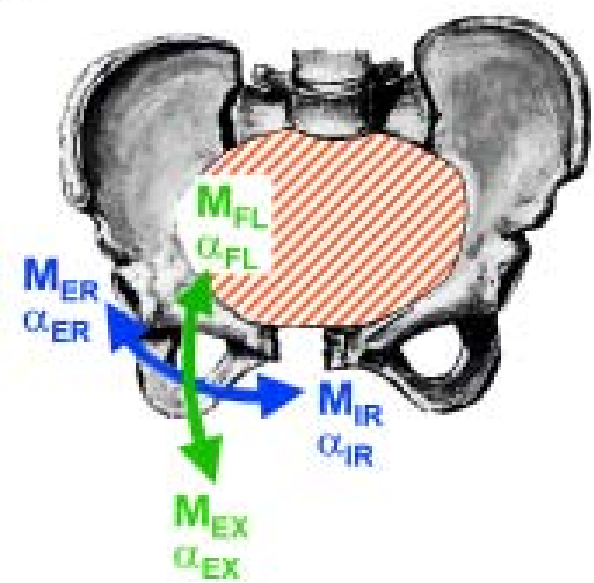

b

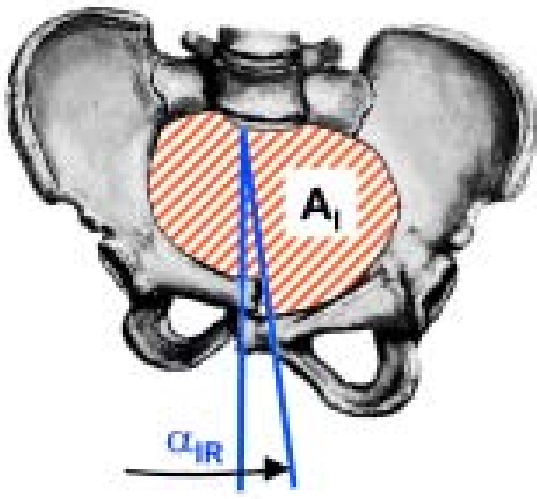

Fig. 3

a, Stability assessment of open-book fractures. Defined rotational stress $\left(\mathrm{M}_{\mathrm{IR} / \mathrm{ER}}, \mathrm{M}_{\mathrm{FL} / \mathrm{EX}}\right)$ induced rotation of the unstable hemipelvis $\left(\alpha_{\mathrm{IR} / \mathrm{ER}}, \alpha_{\mathrm{FL} / \mathrm{EX}}\right) b$, Safety assessment of lateral compression fractures in terms of internal rotation $\left(\alpha_{\mathbb{R}}\right)$ and pelvic inlet area $\left(A_{1}\right)$.
(Synthes, Paoli, Pennsylvania). Stability was assessed in terms of the internal-external rotation and flexion-extension rotation of the unstable hemipelvis in response to defined stress (9-Nm internal-external rotation and flexion-extension moments) (Fig. 3).

Safety Assessment: The potential for harm due to emer- gent application of a pelvic sling to unidentified pelvic fractures was assessed with use of eight human cadaveric full-body specimens. A worst-case scenario was modeled by application of the pelvic sling to unstable Young-Burgess type-II lateral compression fractures. The pelvic sling was applied and tensioned to $180 \mathrm{~N}$ at the greater trochanteric level. Three-
Fig. 4

Sling tension required to achieve complete reduction of the diastasis of the symphysis pubis associated with Young-Burgess type-II and III anteroposterior compression fractures, shown for three distinct levels of application of the pelvic sling.

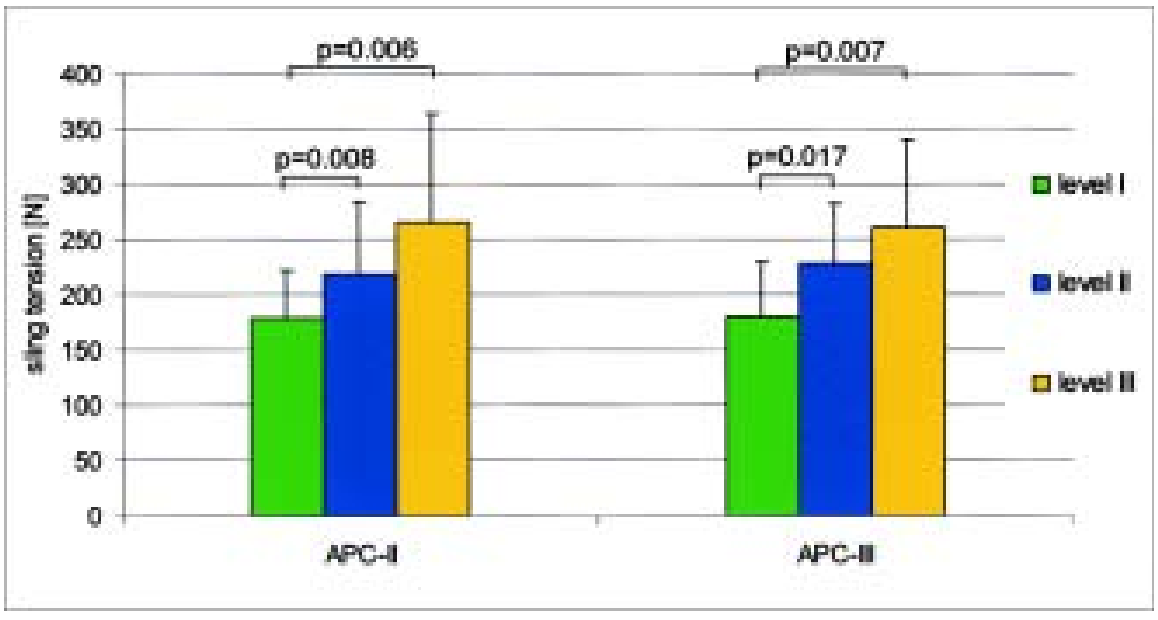




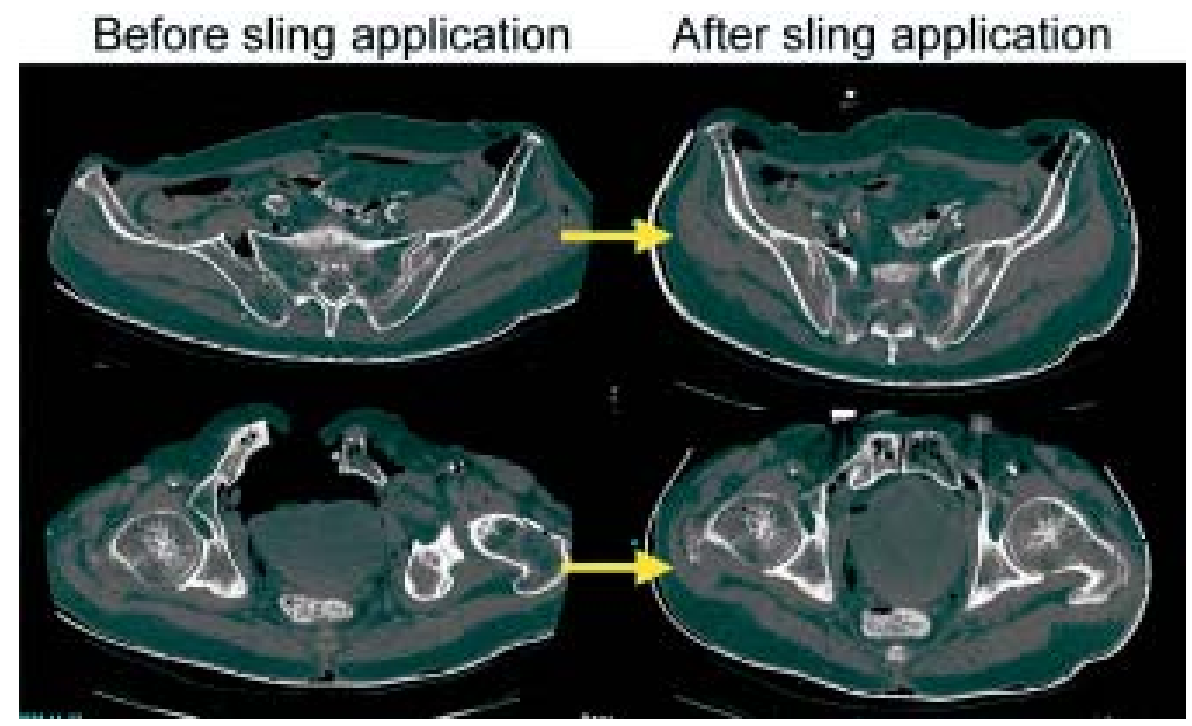

Fig. 5

Young-Burgess type-III anteroposterior compression fracture before and after application of the pelvic sling at level I, demonstrated for one cadaveric specimen.

dimensional-motion tracking sensors were attached to each hemipelvis to assess pelvic ring collapse in terms of the internal rotation of the unstable hemipelvis (Fig. 3). Inlet radiographs were made to measure the corresponding decrease in pelvic inlet area.

\section{Results}

D eduction with use of the pelvic sling required signifiR cantly less tension at level I compared with levels II and III (Fig. 4). At level I, average sling tensions of $177 \pm 44 \mathrm{~N}$ and $180 \pm 50 \mathrm{~N}$ were required to yield complete reduction of typeII and III anteroposterior compression fractures, respectively (Fig. 5).
Application of the pelvic sling significantly stabilized the open-book pelvic fractures, as evidenced by $61 \%$ and $55 \%$ decreases in pelvic instability in response to internal-external rotation and flexion-extension stresses, respectively (Fig. 6). The stability provided by the noninvasive pelvic sling was directly comparable with that provided by the posterior pelvic $\mathrm{C}$ clamp. However, the pelvic sling provided only one-third of the flexion-extension stability and one-tenth of the internalexternal rotation stability as compared with the anteriorly applied external fixator.

Application of the pelvic sling to lateral compression fractures after passive relaxation caused a mild increase in pelvic inlet area and internal rotation to $10.0 \pm 8.2 \%$ and $12.4 \pm$
Fig. 6

Rotational instability of a Young-Burgess type-III anteroposterior compression fracture before and after stabilization with a pelvic sling, a C-clamp, and an external fixator.

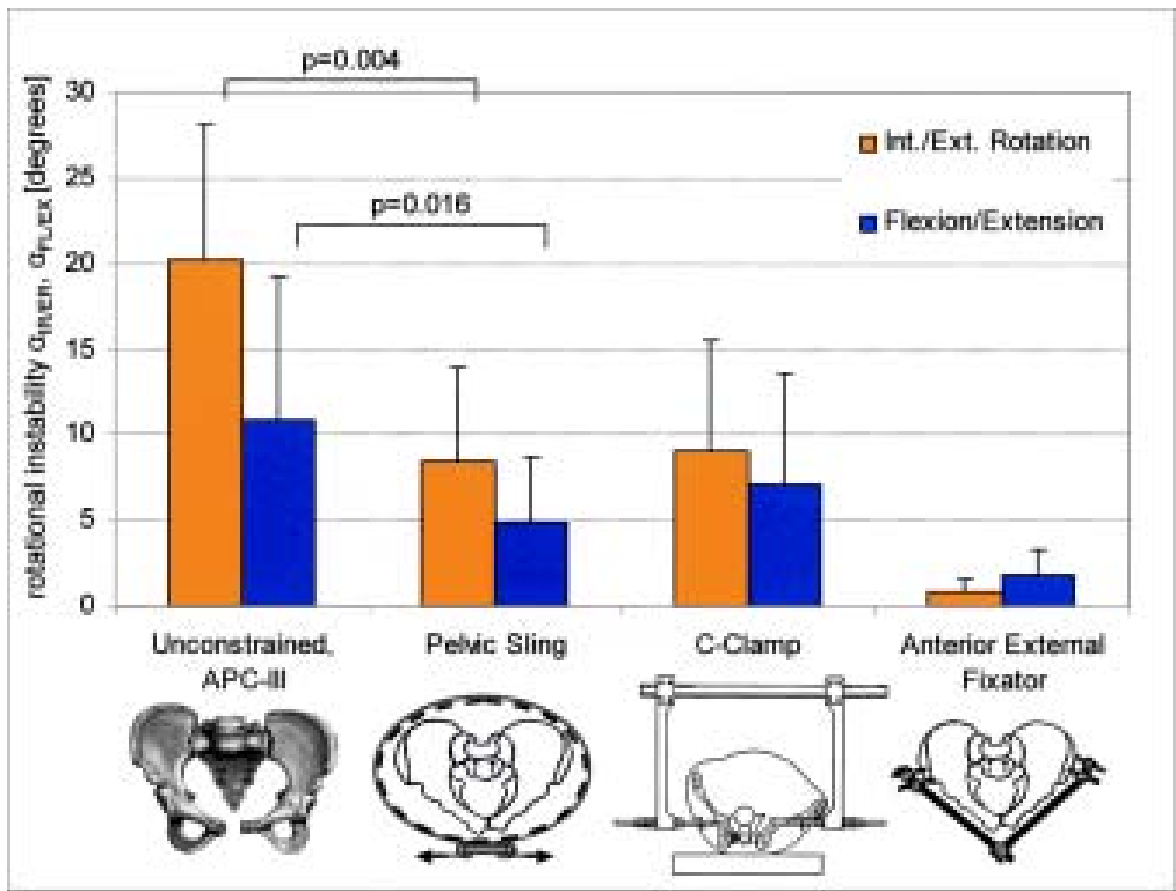


THE JOURNAL OF BONE \& JOINT SURGERY • JBIS. ORG VOLUME 84-A · SuPPLEMENT $2 \cdot 2002$
Emergent Management of Pelvic Ring Fractures With Use of Circumferential Compression

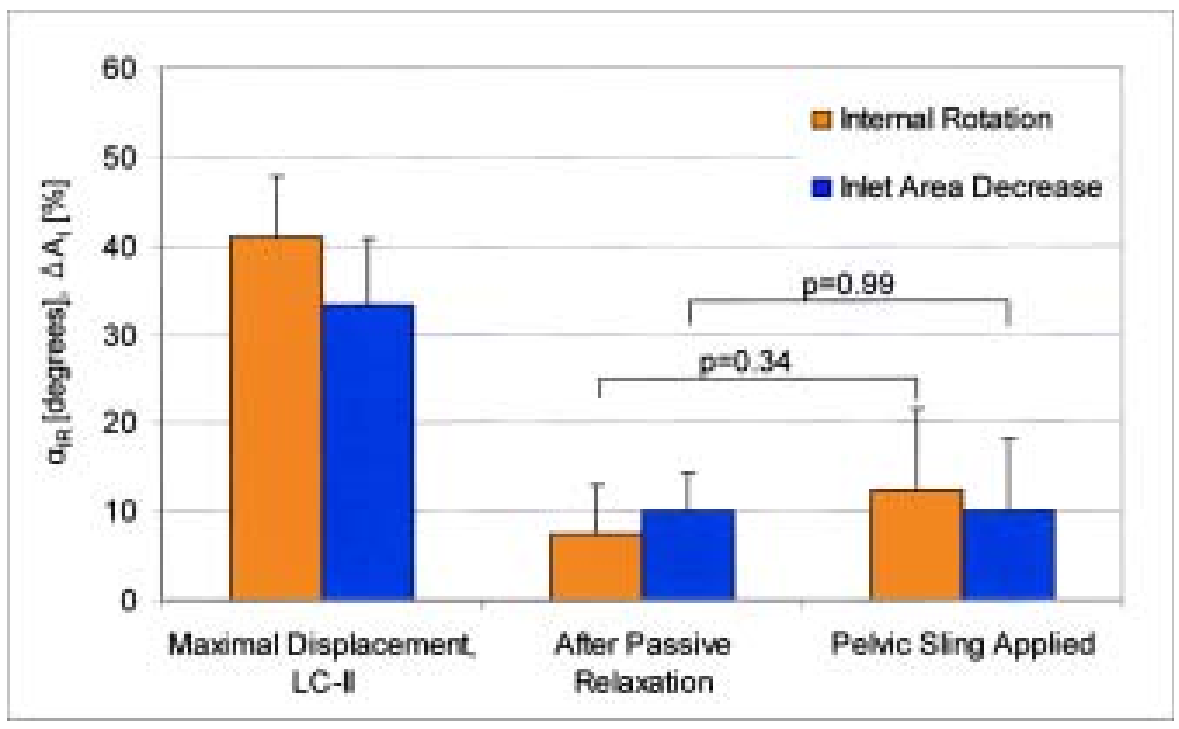

Fig. 7

Pelvic ring collapse of a Young-Burgess type-Il lateral compression fracture in terms of pelvic inlet area decrease $\left(\Delta A_{1}\right)$ and internal rotation $\left(\alpha_{1 R}\right)$ at the time of fracture, after passive relaxation, and after pelvic sling application. $9.1^{\circ}$, respectively (Fig. 7). The increases in pelvic inlet area and internal rotation due to application of the pelvic sling to the relaxed lateral compression fractures were not significant, and the measured values were more than four times smaller than those associated with the maximal displacement observed after fracture creation.

\section{Discussion}

$\mathbf{C}$ arly reduction and stabilization of open-book pelvic frac$\mathbf{D}_{\text {tures is recommended to reduce the risk of life-threatening }}$ hemorrhage $\mathrm{e}^{5-11}$. The results of this study suggest that, for optimal reduction of open-book pelvic fractures, a pelvic sling should be applied around the greater trochanters and the symphysis pubis and should be tensioned to $180 \mathrm{~N}$. The pelvic sling provided significant stabilization, but it provided less stability than an anterior external fixator did. The sling, therefore, is well suited for temporary stabilization of the acutely injured patient prior to and during patient transport or transfer. As the pelvic sling did not cause a significant overreduction of the unstable lateral compression fractures, it may be applied safely at the accident scene before patient transport. This conclusion is based on and limited to a specific and controlled pelvic sling application. Currently, such a pelvic sling with integrated tension control is being used in clinical trials at two level-I trauma centers to document the amount of reduction and stabilization achieved in a controlled clinical setting (Fig. 8).

In conclusion, circumferential pelvic compression by
Fig. 8

Pelvic sling prototype for clinical trial use.

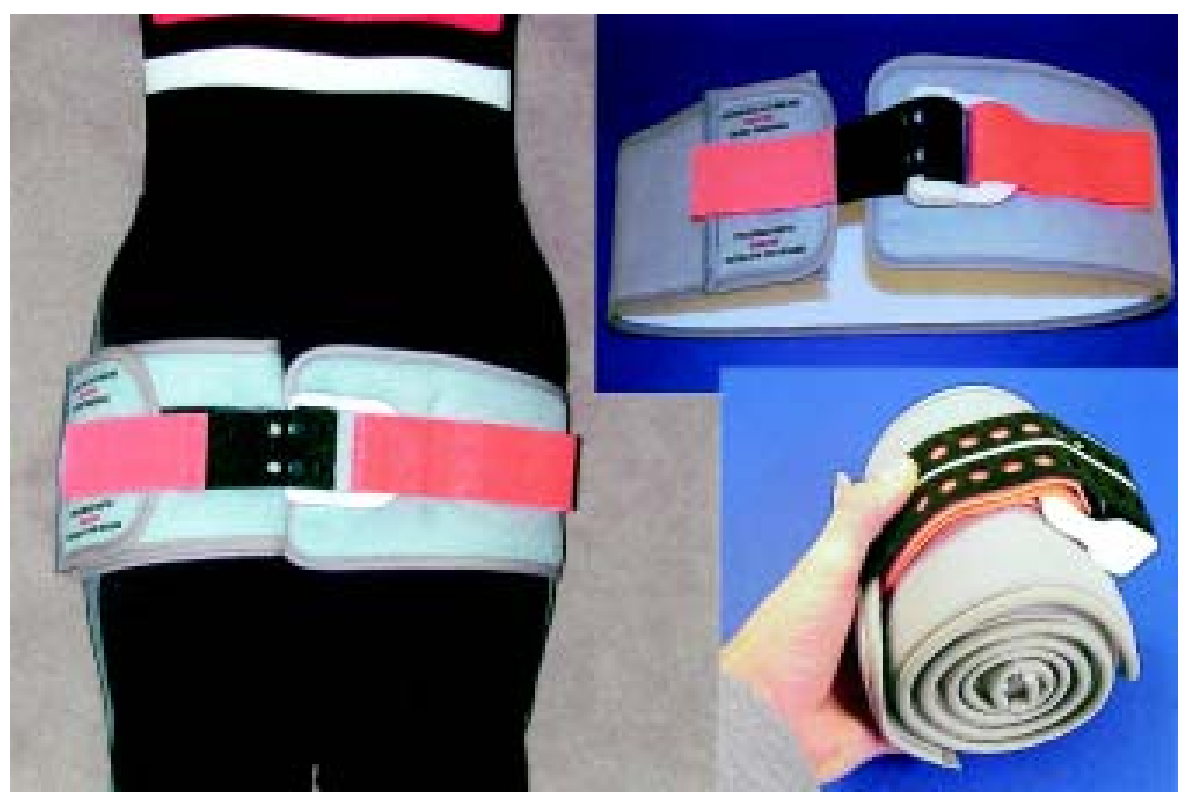


THE JOURNAL OF BONE \& JOINT SURGERY · JBJS.ORG VOLUME 84-A · SUPPLEMENT $2 \cdot 2002$
Emergent Management of Pelvic Ring Fractures WiTH USE OF CiRCUMFERENTIAL COMPRESSION means of the pelvic sling is an effective, noninvasive, and safe stabilization approach that is well suited for emergent, temporary management of open-book pelvic fractures at the accident scene.

NotE: This work was funded by the United States Office of Naval Research and the Legacy Research Foundation.

Corresponding author: Michael Bottlang, $\mathrm{PhD}$

Biomechanics Laboratory, Legacy Health System, Legacy Clinical
Research \& Technology Center, 1225 N.E. 2nd Avenue, Portland, OR 97232. E-mail address: mbottlan@lhs.org

In support of their research or preparation of this manuscript, one or more of the authors received grants or outside funding from Legacy Research Foundation and the U.S. Office of Naval Research (Grant N00014-01-1-0132). None of the authors received payments or other benefits or a commitment or agreement to provide such benefits from a commercial entity. No commercial entity paid or directed, or agreed to pay or direct, any benefits to any research fund, foundation, educational institution, or other charitable or nonprofit organization with which the authors are affiliated or associated.

\section{References}

1. Kregor PJ, Routt ML Jr. Unstable pelvic ring disruptions in unstable patients. Injury. 1999;30(Suppl 2):B19-28.

2. Routt ML Jr, Simonian PT, Ballmer F. A rational approach to pelvic trauma. Resuscitation and early definitive stabilization. Clin Orthop. 1995;318:61-74.

3. Vermeulen B, Peter R, Hoffmeyer P, Unger PF. Prehospital stabilization of pelvic dislocations: a new strap belt to provide temporary hemodynamic stabilization. Swiss Surg. 1999;5:43-6.

4. Simpson T, Krieg JC, Heuer F, Bottlang M. Stabilization of pelvic ring disruptions with a circumferential sheet. J Trauma. 2002;52:158-61.

5. American College of Surgeons. Advanced trauma life support for doctors, ATLS. Instructor Course Manual, 00T-24: 206-09, 1997.

6. Burgess AR, Eastridge BJ, Young JW, Ellison TS, Ellison PS Jr, Poka A Bathon GH, Brumback RJ. Pelvic ring disruptions: effective classification system and treatment protocols. J Trauma. 1990;30:848-56.

7. Dujardin FH, Hossenbaccus M, Duparc F, Biga N, Thomine JM. Long-term functional prognosis of posterior injuries in high-energy pelvic disruption. J Orthop Trauma. 1998;12:145-51.

8. Ganz R, Krushell RJ, Jakob RP, Kuffer J. The antishock pelvic clamp. Clin Orthop. 1991;267:71-8.

9. Grimm MR, Vrahas MS, Thomas KA. Pressure-volume characterization of the intact and disrupted pelvic retroperitoneum. J Trauma. 1998;44:454-9.

10. Meighan A, Gregori A, Kelly M, MacKay G. Pelvic fractures: the golden hour. Injury. 1998;29:211-3.

11. Vrahas MS, Wilson SC, Cummings PD, Paul EM. Comparison of fixation methods for preventing pelvic ring expansion. Orthopedics. 1998;21: 285-9. 